\title{
Performance of a thrombectomy device for aspiration of thrombus with various sizes based on a computational fluid dynamic modelling
}

\author{
Sajjad Soleimani*, Gabriele Dubini, and Giancarlo Pennati \\ Laboratory of Biological Structure Mechanics, Department of Chemistry, Materials and Chemical \\ Engineering "Giulio Natta", Politecnico di Milano, Milan, Italy \\ *Corresponding Author, zzsajad@yahoo.com
}

\begin{abstract}
It is important to thoroughly remove the thrombus within the course of aspiration thrombectomy, otherwise it may lead to further embolization. The performance of the aspiration thrombectomy device with a generic geometry is studied through the computational approach. In order to model the thrombus aspiration, a real left coronary artery is chosen while thrombi with various sizes are located at the bifurcation area of the coronary artery and depending on the size of the thrombus it is stretched toward the side branches. The thrombus occupies the artery resembling the blood current obstruction in the coronary vessel similar to the situation which leads to the heart attack. It is concluded that the aspiration ability of the thrombectomy device is not linked to the thrombus size, it is rather linked to the aspiration pressure and thrombus age (organized versus fresh thrombus), however the aspiration time period correlates to the thrombus size. The minimum applicable aspiration pressure is also investigated in this study.
\end{abstract}

\section{Key words}

Aspiration thrombectomy device, thrombus size, left coronary artery, computational fluid dynamic
Abbreviations
CFD, computational fluid dynamic
AMI, acute myocardial infarction 
PCI, percutaneous coronary intervention

CABG, Coronary Artery Bypass Graft

\section{Introduction}

The damage of vessel or contact of blood cells to a foreign material triggers the hemostatic system and so the thrombus formation is initiated via two mechanisms: platelet system and coagulation cascade [1]. The thrombus formation and lodge in the coronary vessel specifically in the location of plaque buildup in the artery may lead to the blood blockage. This issue should be treated immediately to prevent acute myocardial infarction (AMI).

If the coronary vessel is blocked with thrombus, depending on the emergency, severity, situation and other parameters, several methods are available to tackle the issue: the coronary vessel could be treated with thrombus busting medicines, thrombus removing devices via Percutaneous Coronary Intervention (PCI) and Coronary Artery Bypass Graft (CABG) surgery.

In thrombus busting methods, a pharmacological approach is used to dissolve the thrombus. On the contrary, during the PCI, the thrombus is mechanically removed by means of a device mounted on a catheter, i.e. a very thin tube guided toward the occluded vessel. Removal can occur according to different methods, including breaking down the thrombus and aspirating it. Finally, CABG introduced in 1968 [2] is a conventional and standard approach in many cases. If the patient is well selected, the usage of thrombectomy aspiration device (a type of PCI) is a very reliable approach with minimal clinical drawbacks and high effect in many cases [3]. Via this approach the thrombus is removed only through the aspiration force.

The PCI methods when combined with a completing method such as stenting have an astonishing outcome for patients who suffer from acute ST-segment elevation myocardial infarction (STEMI) [4, 5, 6, 7, 8, 9]. It should be noted that patients who have recently undergone surgery or the patients who had trauma may not be eligible for thrombolysis so the extraction thrombectomy is a good choice for such patients to remove the thrombus [10].

The most important drawback of percutaneous treatments of AMI is their potential for further embolism [11]. It is important that the device would be able to thoroughly remove the thrombus without leaving any debris or part of thrombus non aspirated. Among thrombectomy devices used for PCI method, the aspiration devices are simple to apply [11], which is a great positive 
aspect providing enough means to deal with the emergency situation, typical for the patients who face AMI.

The aim of the current work is to use a numerical approach to give a deeper insight into the aspiration devices' performances. This study is an extension of previous works of our group, exploiting CFD assuming blood and thrombus as two immiscible fluids with different mechanical properties (two-phase flow strategy). Originally, the research had been conducted on the aspiration of thrombus described with a very simple viscosity correlation and considering a simple geometry for the coronary vessel (a straight tube) [12]. Later, a patient specific geometry of vessel representing the main coronary artery was exploited. Moreover the thrombectomy aspiration catheter along with the blood thrombus were located at the bifurcation area of the vessel. A more realistic thrombus mechanical characteristic regarding the viscosity of the thrombus was applied [13]. In the current study we follow the more recent approach [13] and the CFD method is used to investigate the behaviour of thrombectomy aspiration devices in the presence of thrombus with various sizes.

Into the literature, a very few studies focusing on the prediction of the performances of thrombectomy aspiration devices can be found. Furthermore, the literature mostly focused on the cerebral artery thrombectomy, whilst a coronary thrombectmy is presented in the current study. The literature studies were based on either analytical modelling $[14,15,16]$ or experimental tests $[17,18,19]$.

Analytical approaches defect to provide accuracy due to the huge assumed simplifications, thus failing to provide some details of the aspiration phenomenon. As an example, Romero et al. [20, 21] exploited an analytical approach to model the aspiration via a mechanical thrombectomy device by describing the thrombus as a solid block. Their approach fails to predict the thrombus fragmentation. On the contrary, the two-phase flow strategy developed by our group to study the thrombus aspiration allowed us to investigate the performances of catheters with multiple aspirating lumens (central lumen plus lateral holes), observing that the thrombus may fragment and follow various paths [13].

Concerning the experimental investigations, it should be noted that the analysis of various scenarios such as combination of aspiration pressure, thrombus size, thrombus mechanical characteristic and vessel geometry could be very expensive and not easily controllable (e.g. it is 
impossible to maintain the same thrombus properties in different experiments). On the contrary, different scenarios can be easily studied with the help of CFD method.

In this study, the performance of thrombectomy aspiration device applied to aspirate thrombus with various sizes was examined. Possible existence of a critical pressure is investigated under which the aspiration process may fail to remove thrombus thoroughly.

\section{Material and methods}

The whole system of an aspiration thrombectomy device consists of several components, including syringe, stop cock valve, luer lock connectors, lumen, median part and tip of the catheter. After the opening of the stopcock valve, the negative pressure generated into the syringe will propagate in the entire device and into the blocked coronary vessel. It is worth noting that, during the procedure, the applied vacuum gradually decays down to zero due to filling the syringe (the source of aspiration pressure).

Modelling of the whole system of aspiration thrombectomy device is very expensive and unsuitable. Hence, only the tip of a generic representative device was modeled (Figure 1) and located close to the obstructed region of LCA while an appropriate negative pressure value is applied at its outlet (Figure 2).

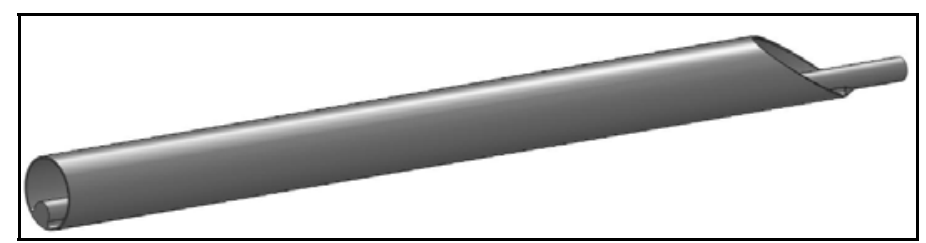

Figure 1: The tip of thrombectomy device with the length of $23 \mathrm{~mm}$ and outer diameter of $1.72 \mathrm{~mm}$

Coronary artery consists of the left coronary artery (LCA) and the right coronary artery. In general, the left coronary artery has two main branches of anterior interventicular and a circumflex branch [22].To model the aspiration of thrombus in the realistic coronary geometry, a patient specific bifurcated segment of LCA was exploited. The geometry model (courtesy of the European Bifurcation Club, Lisbon, 2011) was reconstructed on the basis of IntraVascular 
UltraSound and Computed Tomography techniques. The details of this method can be found in Giessen et al. [23].

The thrombus is assumed to be located at the bifurcation area and to fill part of the vessel; a complete vessel blockage was then created similar to the condition of a patient at AMI event. The tip of the thrombectomy device is positioned very close to the blood thrombus, without touching it. The main branch of the coronary artery was extended to create a model region around the tip.

Depending on the volume of modelled thrombus, part of daughter branches may be blocked as well (Figure 2). Since thrombus volumes observed in the coronary arteries are quite variable [16, $20,21,24,25]$, in this study four different thrombus volumes (56, 67, 80 and $95 \mathrm{~mm}^{3}$ ) were modeled.

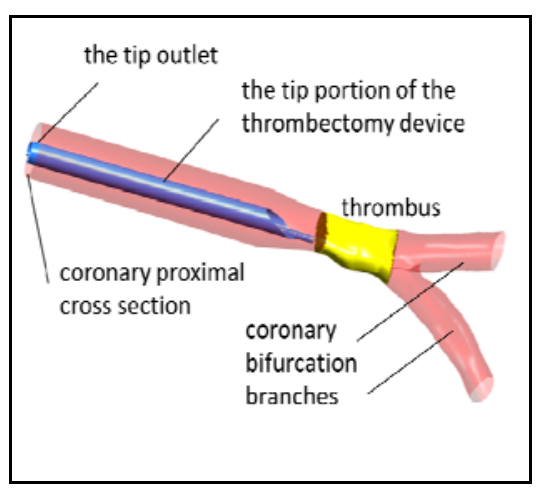

(A)

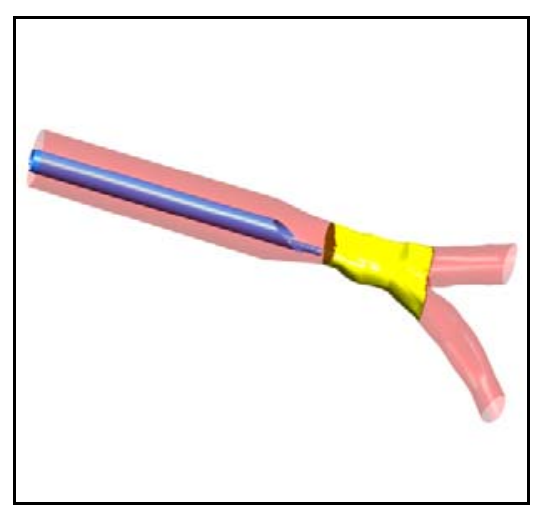

(C)

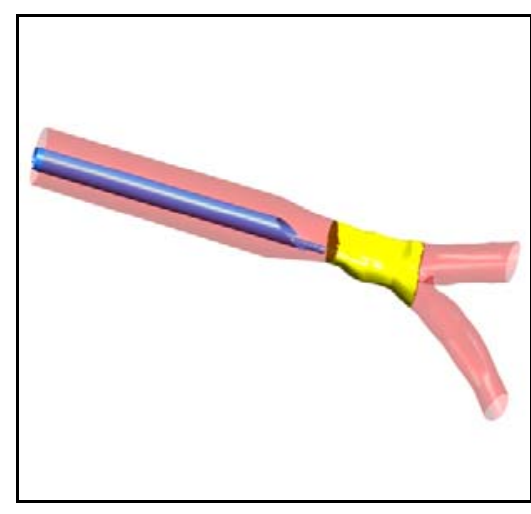

(B)

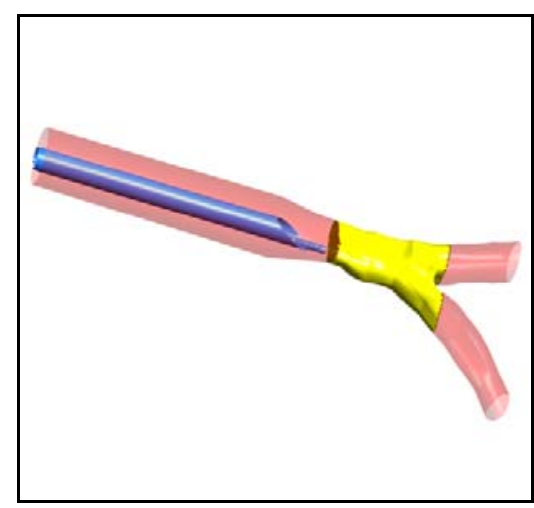

(D)

Figure 2: The initial position of thrombus (yellow color) with different sizes (from 56 to $95 \mathrm{~mm}^{3}$ ). Panel (A) shows also the different parts of the model 
In order to model the thrombus aspiration, a two phase flow approach was applied following our previous investigations [13]. Namely, blood and thrombus were considered as two nonNewtonian immiscible fluids with quite different rheological parameters.

The non-Newtonian blood rheology was modelled using the Bird-Carreau model [26, 27]:

$\mu=\mu_{\infty}+\left(\mu_{0}-\mu_{\infty}\right)\left(1+\lambda^{2} \dot{\gamma}^{2}\right)^{\frac{n-1}{2}}$

where $\mu_{0}=0.56$ Pa.s, $\mu_{\infty}=0.0345$ Pa.s,$\lambda=3.313$ s and $n=0.3568$

A reasonable thrombus rheology was derived from experimental tests specifically performed [28]. Hence, thrombus was modelled as a non-Newtonian fluid with very high viscosity described by a power law equation:

$\eta(\dot{\gamma})=A \times \dot{\gamma}^{(-B)}$

where A and B are the coefficients reported in Table 1 for three different thrombi with different viscosities [13]. Using these parameter values, the viscosity of fresh thrombus is about 50-200 times that of blood, while the viscosity of organized thrombus is about 1000 times the blood viscosity. Moreover, a surface tension of $0.15 \mathrm{~N} / \mathrm{m}$ was introduced to better model thrombus response during the thrombectomy aspiration [13].

Table 1: Viscosity coefficients for different blood thrombi

\begin{tabular}{|c|c|c|}
\hline Case & A & B \\
\hline Fresh thrombus - Low viscosity (LV) & 21.45 & 0.8866 \\
\hline Fresh thrombus - Medium viscosity (MV) & 34.14 & 0.9188 \\
\hline Organized thrombus - High viscosity (HV) & 98.65 & 0.9552 \\
\hline
\end{tabular}

The interface locations between the two fluids (blood and thrombus) are traced through Volume of Fluid (VOF) approach. In VOF methodology the volume of each fluid $\left(\mathrm{V}_{\text {fluid }}\right)$ in the computational cell is defined through the formula $\mathrm{V}_{\text {fluid }}=\gamma \mathrm{V}_{\text {cell }}$ in which $\mathrm{V}_{\text {cell }}$ is the volume of each cell. When the cell is totally filled by one fluid, $\gamma$ is equal to 1 ; if it is filled by the other fluid, $\gamma$ is equal to 0 ; if the cell is partially filled by either volume, $\gamma$ should satisfy the following equation:

$\frac{\partial \gamma}{\partial \mathrm{t}}+\nabla(\gamma \mathrm{u})+\nabla(\gamma(1-\gamma) \mathrm{u})=0$ 
Where, $\mathrm{u}$ is the velocity field at the interface of two fluids. A single momentum equation is solved for the entire domain and the velocity field is shared between the fluid phases. The properties in each cell $(\theta)$ is computed according to the following equation:

$\theta=\gamma \theta_{\text {fluid } 1}+(1-\gamma) \theta_{\text {fluid2 }}$

The thrombus removal occurs in the two stages; at the first stage the thrombus should be detached from the vessel wall followed by the second stage which is the aspiration of bulk of thrombus through a very thin tube (PCI catheter). In this study the performance of thrombus aspiration at the second stage is investigated considering the interaction between thrombus and wall is due to the no-slip boundary condition and to the thrombus viscosity by affecting the shear gradient at the wall.

To study the thrombus movement and aspiration, the CFD simulations (finite volume) were done by using ANSYS ICEM CFD 12.1 (ANSYS Inc., Canonsburg, PA, USA). The thrombus aspiration phenomenon was described as a laminar and unsteady flow. Thrombus movement towards the tip of the thrombectomy device was caused by applying a pressure difference between the coronary proximal cross section, bifurcation branches and the tip outlet. The aspiration pressure varies within the range of 15 to $60 \mathrm{kPa}$. The adopted aspiration pressures are within the range of the pressure used in such devices [12, 14, 15].

The numerical domain was discretized via the tetrahedral type mesh while the area close to the wall of the catheter is discretized via the prism mesh. The tetrahedral mesh needs much less effort to create and the prism mesh close to the wall of the catheter provides more accurate result. Both grid and time step sensitivities were performed to ensure the reliability of the numerical model adopting the aspirated thrombus volume as the controlled variable. According to the sensitivity analysis, a final mesh of about 1 millions of elements and a time step of $0.01 \mathrm{~s}$ were adopted for the simulations [13].

The residual values of continuity, momentum and volume of fluid were considered to drop to the level of $5 \times 10^{-5}, 5 \times 10^{-5}$ and $5 \times 10^{-4}$, respectively.

Two sets of simulation were performed (Table 2). First, the influence of thrombus size on the thrombectomy device performance was evaluated for a low aspiration pressure $(20 \mathrm{kPa})$ and considering a fresh thrombus with low viscosity. Second, the influence of different aspiration 
pressures was assessed for all the considered thrombus viscosities, for a fixed thrombus volume, $56 \mathrm{~mm}^{3}$.

Table 2. Sets of simulation for scenarios based on the various thrombus size, aspiration pressure and thrombus viscosity

(A)

\begin{tabular}{|c|c|c|c|}
\hline case & Thrombus size $\mathrm{mm}^{3}$ & Pressure $\mathrm{kPa}$ & Viscosity Type \\
\hline 1 & 56 & 20 & LV \\
\hline 2 & 67 & 20 & LV \\
\hline 3 & 80 & 20 & LV \\
\hline 4 & 95 & 20 & LV \\
\hline
\end{tabular}

(B)

\begin{tabular}{|c|c|c|c|}
\hline case & Thrombus size $\mathrm{mm}^{3}$ & Pressure $\mathrm{kPa}$ & Viscosity Type \\
\hline 5 & 56 & 15 & LV \\
\hline 6 & 56 & 20 & LV \\
\hline 7 & 56 & 30 & LV \\
\hline 8 & 56 & 40 & LV \\
\hline 9 & 56 & 50 & LV \\
\hline 10 & 56 & 60 & LV \\
\hline 11 & 56 & 15 & MV \\
\hline 12 & 56 & 20 & MV \\
\hline 13 & 56 & 30 & MV \\
\hline 14 & 56 & 40 & MV \\
\hline 15 & 56 & 50 & MV \\
\hline 16 & 56 & 60 & MV \\
\hline 17 & 56 & 15 & HV \\
\hline 18 & 56 & 20 & HV \\
\hline 19 & 56 & 30 & HV \\
\hline 20 & 56 & 40 & HV \\
\hline 21 & 56 & 50 & HV \\
\hline 22 & 56 & 60 & HV \\
\hline
\end{tabular}

\section{Results and discussion}


The general behavior of aspiration of thrombus via thrombectomy devices had been discussed in our previous works $[13,29]$ : the aspiration process passes through three phases. In the first phase, the speed of thrombus aspiration is very slow, since the thrombus is gradually starting to move from its position. Conversely, in the second phase, the thrombus is aspirated very rapidly and the main bulk of thrombus moves toward the catheter and crosses the tip lumen. Finally, during the third phase, the aspirated fluid is mostly blood, with trivial quantities of thrombus; hence, the aspirated thrombus increases very slowly and, in some cases, tends to an asymptotic value lower than the whole thrombus volume, thus in some situations the thrombus cannot be fully aspirated from the vessel.

The effect of thrombus size and applied aspiration pressure on the level of aspirated thrombus volume and the time period of such procedure is discussed here.

In the current study the thrombus is initially lodged at the bifurcation region of the LCA model. Four different sizes of thrombus were investigated, with increasing value so that the thrombus gradually extends within the vessel branches. The idea of studying on such scenarios is to measure the differences between the aspiration performance: level of aspiration and the essential time period for various thrombus sizes. As shown in Figure 3, it is interesting to note that the thrombus size and even penetration to the branches does not influence the overall aspirated thrombus volume, only the time period of aspiration increases.

It might be noted that the aspiration thrombectomy devices usually use the syringe for both pressure source and reservoir in order to gather the aspirated content: blood and thrombus, which causes some limitations. First, there is a limited capacity for the aspirated content, thus by increasing the aspiration time the reservoir will be filled by the blood, preventing the aspiration of thrombus. Secondly, by gradually filling the syringe with blood and part of the thormbus, the aspiration pressure declines very sharply. Such limitations suggest that the slow thrombus aspiration may increase thrombus aspiration failure in real life surgery. With respect to the later fact, increasing the size of thrombus negatively affects the thrombectomy efficiency.

Actually, during the intervention, the clinicians can slightly move the catheter tip back and forth in order to aspirate the remaining thrombus. For the sake of simplicity (it requires a moving boundary simulation), this effect was not simulated in the current study. 


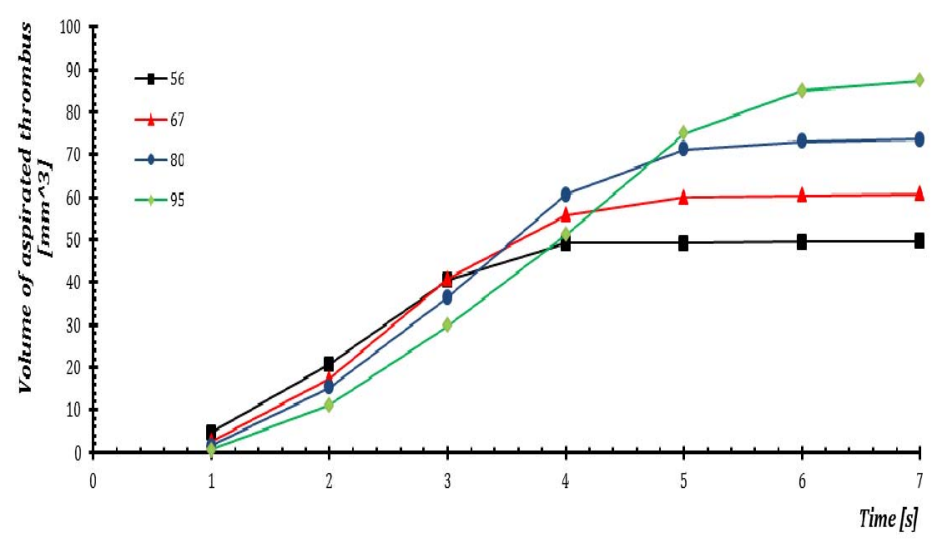

(A)

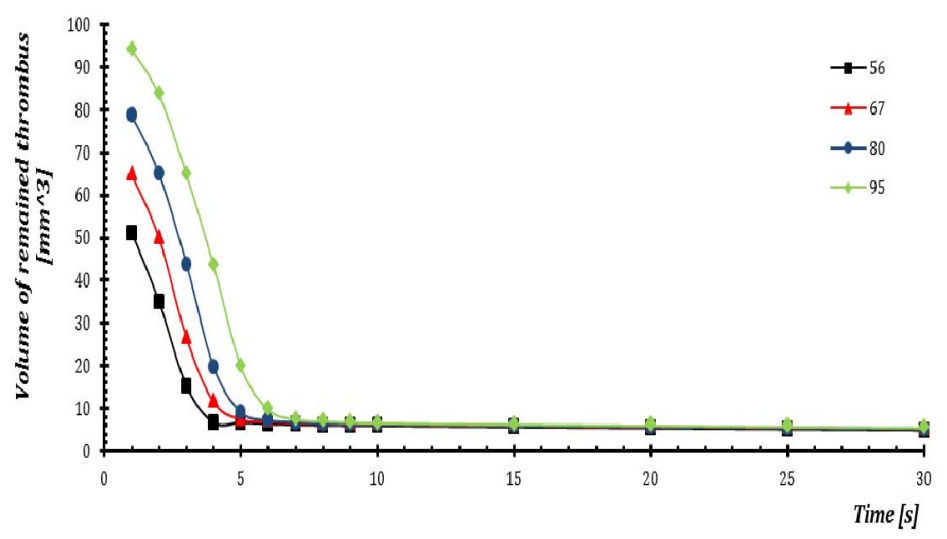

(B)

Figure 3: A) Volume of aspirated thrombus and B) volume of remained thrombus versus time: the thrombus initial volume is 56, 65, 80 and $95 \mathrm{~mm}^{3}$

The combination of aspiration pressure and thrombus viscosity are two parameters which significantly affect the thrombus aspiration performance on such single hole thrombectomy device (Figures 4 to 7). Within the current study, the range of pressure varied widely; from 15 $\mathrm{kPa}$ (around the minimum of left ventricular systolic pressure) at the lowest to $60 \mathrm{kPa}$ (more than half of maximum tolerable pressure by vessel - $100 \mathrm{kPa}$ [30]) at the highest level. The chosen wide range of aspiration pressure will cover various scenarios for our study.

By applying the low aspiration pressure, part of thrombus remains non aspirated and becomes more critical when the thrombus viscosity increases. It seems that a pressure with critical value can be introduced as a threshold. Above the threshold it is guaranteed that the thrombus can be thoroughly aspirated and the difference is just the matter of time period. The aspiration pressure 
less than $30 \mathrm{kPa}$ shows a critical value. Under such level of aspiration a considerable amount of thrombus is not aspirated. The worse situation is related to the highest viscosity at which no thrombus is aspirated within the first 40 seconds of operation(Figure 6B).

The role of pressure is highlighted when the aspiration of more organized thrombus is of the purpose. In that situation the higher pressure value significantly decreases the overall time period for thrombus aspiration. Although high aspiration pressure is beneficial, it is restricted by physiological parameters and cannot exceed the vessel tolerance.

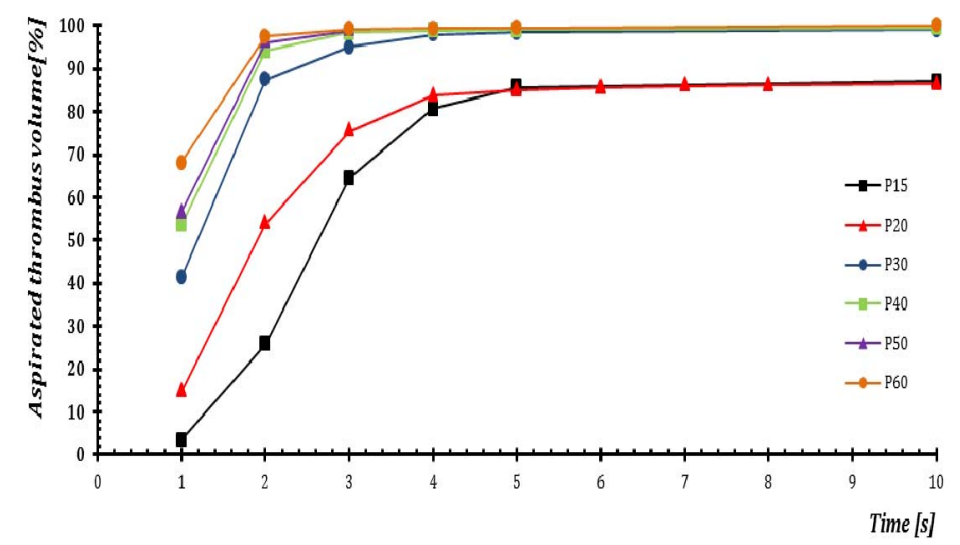

Figure 4: Percentage of aspirated thrombus volume with low viscosity

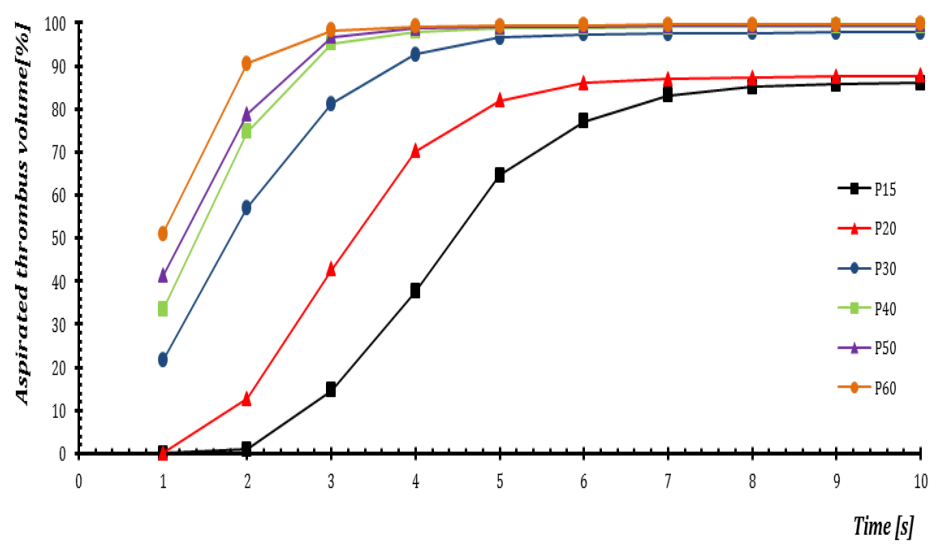

Figure 5: Percentage of aspirated thrombus volume with medium viscosity 


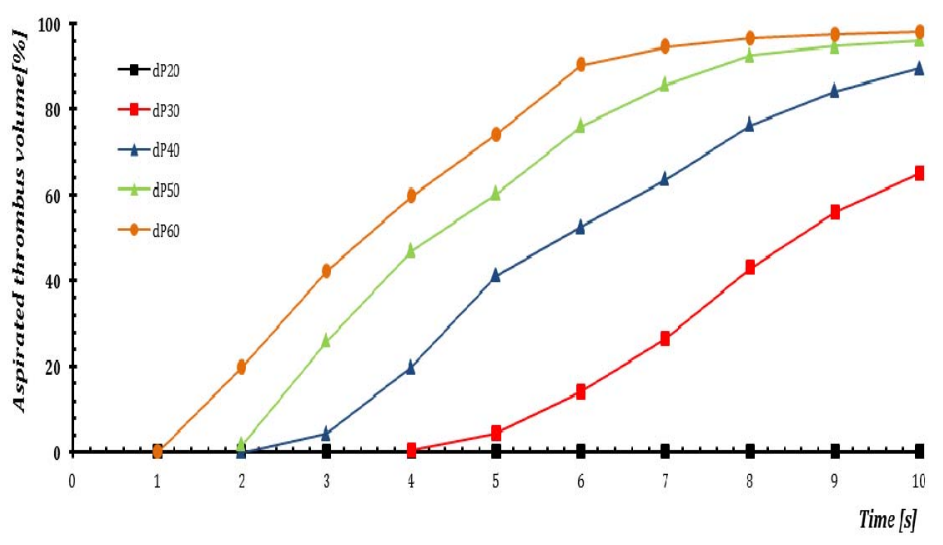

(A)

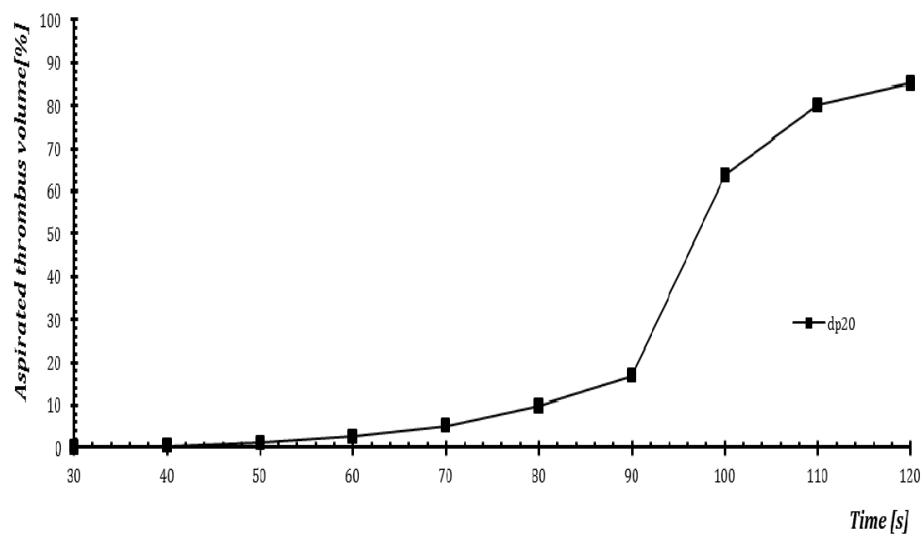

(B)

Figure 6: Percentage of aspirated thrombus volume with high viscosity. A) 20 to $60 \mathrm{kPa}$ aspiration pressure, B) 20 $k P a$ aspiration pressure

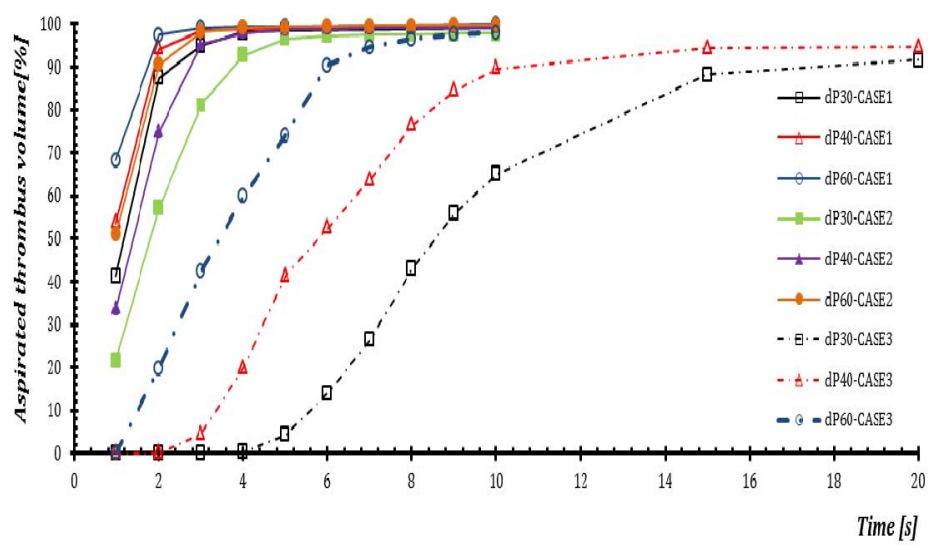

Figure 7: Percentage of aspirated thrombus volume with low, medium and high viscosity 


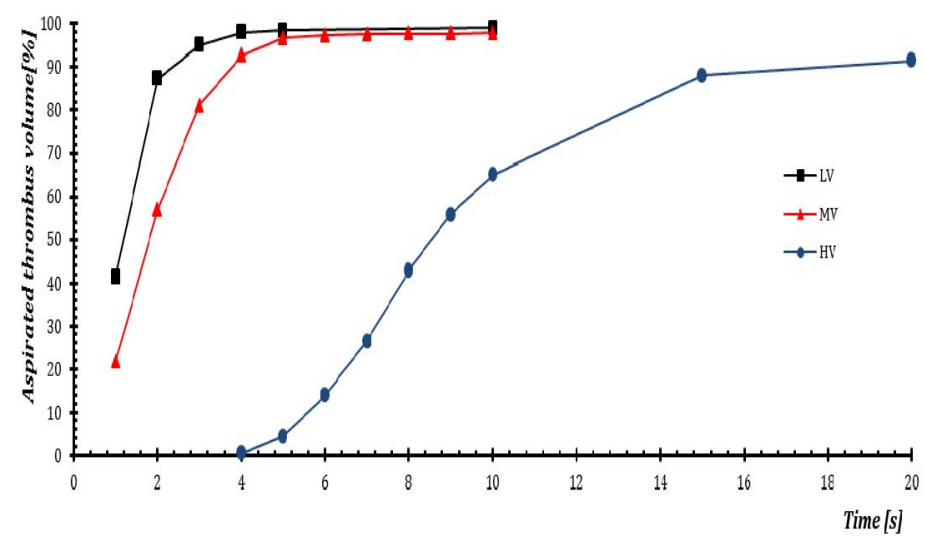

Figure 8: Percentage of aspirated thrombus volume for the pressure with critical value (30 $\mathrm{kPa}$ ) and thrombus with low, medium and high viscosity

Figure 8 indicates the aspiration behavior while the aspiration pressure of $30 \mathrm{kPa}$ is applied. Within 20 seconds, the device can aspirate around 95\% of thrombus with the highest viscosity level. At the first stage of this study (Figure 3), in order to examine the ability of the device to aspirate thrombus with various sizes in the critical situations, the pressure value $20 \mathrm{kPa}$ which is lower than the threshold was applied.

\section{Limitations of the study}

Our study is based on a patient specific model of the LCA. Although a range of LCA models extracted from various patients are essential to be studied for a solid conclusion, it is believed that our preliminary study initiates a discussion on the application of aspiration thrombectomy for thrombus with various sizes. Moreover this study could be applicable on the LCA with nearly similar geometrical characteristic.

Viscoelastic parameters of thrombus were initially derived from our previous work and converted to a viscous parameter [28] to facilitate the computational modeling. For a more realistic conclusion a direct viscoelastic model of thrombus (considering both viscosity and elasticity) could be used. Moreover the organized thrombus may have a more complicated behavior due to the non-homogenous consistency originated from the fibrinogen which could be the aim of further study. 
The boundary condition and in specific the applied aspiration pressure is taken constant. In reality the syringe is the source of aspiration which is filled with the aspirated content over time. Thus considering a declining pressure at the aspiration boundary condition as well as the limited time period for the aspiration would be beneficial. However the initial scope of our study is to merely focus on the effect of aspiration on various thrombus sizes. As the above mentioned constraint (declining the pressure over time) is a technological matter that relates to the design of the aspiration thrombectomy device, it might be addressed when the improvement design of such devices is under investigation.

Our study is performed on a general tip shape aspiration thrombectomy device. A comprehensive study on the tip with extra hole design is performed in our previous study [13] for the thrombus with single size. The effect of those designs (thrombectomy devices with extra hole) could be also applicable here to investigate the aspiration behavior on thrombus with various sizes.

The main purpose of this study is to qualitatively investigate the trend of aspiration through thrombectomy device as well as providing a basis for comparison of the aspiration efficiency between different scenarios. With this regard we discarded the attempts to precisely match the simulated quantity of time period to the real life operation which could be a purpose of further study.

The performance of PCI thrombectomy through aspiration catheter is studied while the interaction between wall and the thrombus is non slip. Thus our study presents the details of thrombus aspiration at the second stage, considering that due to the impact of thrombus to the catheter tip, it may partially break and remain non aspirated because of the beveled shape of the PCI catheter tip. Although the attachment of the thrombus to the vessel well is less critical compared to the attachment of plaque in stenosis, it should be further included in the thrombus aspiration modelling to provide more details of thrombectomy application.

Depending on thrombus size and applied pressure, the aspiration of thrombus in our study is in the range of few seconds to few minutes, these values are in accordance with literature although the only reported data found, refer to somehow a different condition [14, 31]. Nevertheless, further study might be conducted to monitor details of thrombus aspiration processes such as thrombus break up, experimentally. While in vivo study of the dynamic details of thrombus aspiration needs a lot of effort, the correct ex-vivo set up is also challenging. One benefit of numerical study is to provide such details in 3-D with much less effort although with the cost of 
less reliability. Moreover the purpose of our study is more related to a qualitative study on thrombus aspiration by providing some preliminary insights in aspiration process and providing a tool for comparison on different scenarios. A very detailed quantitative result will be obtained by ex-vivo setup. Interestingly, our numerical modelling provides some essential hints for such set up.

\section{Conclusion}

The applicability of aspiration thrombectomy for various sizes of thrombus lodged in a patient specific LCA is studied through computational modelling. It is concluded that only the time period of aspiration is affected by thrombus size, instead the ability of aspiration thrombectomy devices are independent from the initial size of thrombus.

Both of above mentioned parameters: "essential aspiration time period" and "remained non aspirated part of thrombus" are crucial points. The "essential aspiration time period" is important due to the technical limitations of aspiration thrombectomy devices: the pressure source declines over time and the reservoir may be rapidly filled with undesired content, i.e. blood instead of thrombus hence providing a relatively short period of time for thrombus aspiration in the real life surgery. The "remained non aspirated part of thrombus" will cause even a more significant issue related to further embolization. Such embolization issue is also discussed and concerned seriously in the literature $[3,18]$.

General behavior of aspiration with three phases of slow aspiration, rapid aspiration and slow aspiration remains similar in all cases. Various values of aspiration pressure when applied to aspirate thrombus with various viscosity will alter time period as well as the level of non aspirated thrombus percentage. According to the applied scenarios in this study, a pressure less than $30 \mathrm{kPa}$ may be considered a critical value, as a considerable part of thrombus in not aspirated for all the modelled cases. A more comprehensive study is however essential to firmly draw a correlation between aspiration pressure value and non-aspirated percentage of thrombus. As mentioned earlier, the percentage of non-aspirated thrombus is of great interest for any study relating to the thrombectomy procedure due to the cause of further embolization and so leading to cardiovascular disease event. 


\section{Acknowledgement}

This research is funded by the European Commission, through the MeDDiCA ITN (grant agreement PITN-GA-2009-238113)

\section{Reference}

[1] Verbiest BCH. Thrombus formation in aneurysms: an experimental study, Division of Cardiovascular Biomechanics. Eindhoven University of Technology, 2008.

[2] Favaloro RG. Saphenous vein autograf replacement of severe segmental coronary artery occlusion: operative technique. The Annals of Thoracic Surgery 1968; 5: 334-9.

[3] Costopoulos C, Gorog DA, Mario CD, Kukreja N. Use of thrombectomy devices in primary percutaneous coronary intervention: A systematic review and meta-analysis. Int J Cardiol 2011; 163: 229-241.

[4] Serruys PW, Morice MC, Kappetein AP, et al. Percutaneous Coronary Intervention versus Coronary-Artery Bypass Grafting for Severe Coronary Artery Disease. The New England Journal of Medicine 2009; 360 (10).

[5] Vink A, Kramer MC, Li X, et al. Clinical and Angiographic Predictors and Prognostic Value of Failed Thrombus Aspiration in Primary Percutaneous Coronary Intervention. Cardiovascular Interventions 2011; 4: 634-642.

[6] Schomig A, Mehilli J, Waha A, Seyfarth M, Pache J, Kastrati A. A Meta-Analysis of 17 Randomized Trials of a Percutaneous Coronary Intervention-Based Strategy in Patients With Stable Coronary Artery Disease, Journal of the American College of Cardiology 2008; 52(11): 894-904.

[7] Svilaas T, Vlaar PJ, Horst IC, et al. Thrombus Aspiration during Primary Percutaneous Coronary Intervention. The new england journal of medicine 2008; 358: 557-567.

[8] Zijlstra F, Hoorntje JC, Boer MJ. Long-term benefit of primary angioplasty as compared with thrombolytic therapy for acute myocardial infarction. The New England Journal of Medicine 1999; 341: 1413-9.

[9] Silber S, Albertsson P, Aviles FF. Guidelines for percutaneous coronary interventions: the Task Force for Percutaneous Coronary Interventions of the European Society of Cardiology. European Heart Journal 2005; 26: 804-47. 
[10] Kucher N, Windecker S, Banz Y, et al. Percutaneous Catheter Thrombectomy Device for Acute Pulmonary Embolism: In Vitro and in Vivo Testing. Radiology 2005; 236: 852-858.

[11] Sakurada M, Ikari Y, Isshiki T. Improved Performance of a New Thrombus AspiraCatheter: Outcomes From In Vitro Experiments and Case Presentation. Catheterization and Cardiovascular Interventions 2004; 63: 299-306.

[12] Pennati G, Balossino R, Dubini G, Migliavacca F. Numerical Simulation of Thrombus Aspiration in Two Realistic Models of Catheter Tips. Artificial Organs 2010; 34: 301-310.

[13] Soleimani S, Dubini G, Pennati G. Possible benefits of catheters with lateral hole on coronary thrombus aspiration: a computational study for different clot viscosity and vacuum pressure. Artificial Organs 2014; 38: 845-55

[14] Romero G, Martinez M, Pearce G, Mera M. An investigation into the performance of a new mechanical thrombectomy device using bond graph modeling: application to the extraction of blood thrombuss in the middle cerebral artery. Simulation: Transactions of the Society for Modeling and Simulation International 2012; 89: 381-391.

[15] Romero G, Martinez ML, Felez J. Applicability of the GP Device to the Circle of Willis Arteries by Using a Mathematical Model. Proceeding of Computer Modelling and Simulation. Cambridge UK 2011.

[16] Pearce G, Perkinson RND, Wong J, et al. The “'GP”' Mechanical Thrombectomy Device. J Stroke Cerebrovasc Dis 2009; 18: 288-293.

[17] Hong-bing Y, WANG J, Nan L, et al. Diver CE versus Guardwire Plus for thrombectomy in patients with inferior myocardial infarction: a trial of aspiration of thrombus during primary angioplasty for inferior myocardial infarction. Chinese Medical Journal 2007; 120 (7):557-561.

[18] Chant H, Ashleigh R, McCollum C. Thrombectomy for Acute Internal Carotid Thrombosis: Five Thrombectomy Devices Compared. Eur J Vasc Endovasc Surg 2004; 27: 403-408.

[19] Sakurada M, Ikari Y, Isshiki T. Improved Performance of a New Thrombus Aspiration Catheter: Outcomes From In Vitro Experiments and a Case Presentation. Catheterization and Cardiovascular Interventions 2004; 63: 299-306

[20] Romero G, Martinez ML, Felez J, Pearce G, Perkinson ND. Analysis and design of a thrombectomy device by using simulation techniques. Proceedings of the IMProVe 2011 International conference on Innovative Methods in Product Design Venice, Italy June 15th 17th, 2011.

[21] Romero G, Martinez ML, Felez J, Pearce G, Perkinson ND. Applicability of the GP Device to the Circle of Willis Arteries by Using a Mathematical Model. 13th International Conference on Modeling and Simulation 2011. 
[22] Snell RS. Clinical Anatomy by Regions. 8th ed. Wolters Kluwer, Lippincott Williams \& Wilkins.

[23] Giessen AG, Schaap M, Gijsen FJH, Groen HC, Walsum T, Mollet NR. 3D fusion of intravascular ultrasound and coronary computed tomography for in-vivo wall shear stress analysis: a feasibility study. Int J Cardiovasc Imaging 2010; 26: 781-796.

[24] Walvick RP, Bratane BT, Henninger N, et al. Visualization of Thrombus Lysis in a Rat Embolic Stroke Model: Application to Comparative Lytic Efficacy. Stroke 2011; 42:1110-5.

[25] Blanc R, Pistocchi S, Babic D, et al. Intravenous flat-detector CT angiography in acute ischemic stroke management. Interv Neuroradiol 2012; 54: 383-391.

[26] Cho YI, Kensey KR. Effects of the non-Newtonian viscosity of blood on flows in a diseased arterial vessel, Part 1: steady flows. Biorheology 1991; 28: 241-262.

[27] Johnston BM, Johnston PR, Corney S, Kilpatrick D. Non-Newtonian blood flow in human right coronary arteries: steady state simulations. J Biomech 2004; 37: 709-720.

[28] Soleimaniamiri S. Coronary thrombus removal by means of aspiration catheter: a numerical and experimental fluid dynamic study (dissertation). Politecnico di Mialno; 2013.

[29] Soleimani S, Pennati G, Dubini G. Effect of Geometrical Characteristic and Clot mechanical Properties on Coronary Clot Aspiration Catheter Performance. Medical Devices Design in Cardiovascular Applications 2013; 254- 281.

[30] Romero G, Martinez ML, Maroto J, Felez J. Modelling and simulation of a thrombectomy probe applied to the middle cerebral artery by using the bond graph technique. Proceedings of the 2010 Spring Simulation Multiconference. SpringSim 2010, Florida USA April 11-15 2010.

[31] Muller-Hulsbeck S, Bengard C, Schwarzenberg H, Gluer CC, Heller M. In vitro effectiveness study of three hydrodynamic thrombectomy devices. Radiology 1999;211:433-9. 